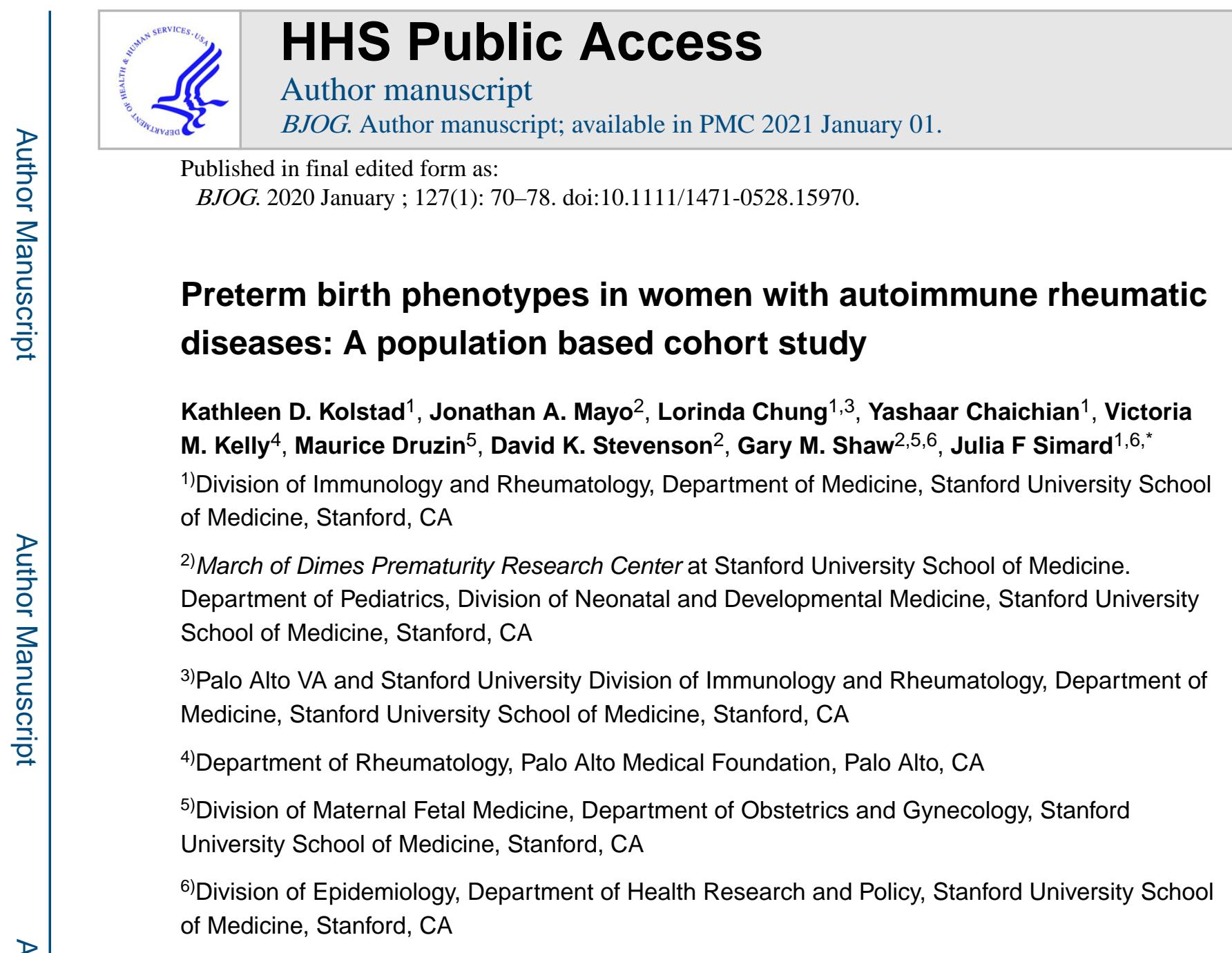

\title{
Abstract
}

Objective: To investigate preterm birth (PTB) phenotypes in women with different autoimmune rheumatic diseases in a large population-based cohort.

Design: Retrospective cohort study.

Setting: California, USA.

Population: All live singleton births in California between 2007 and 2011 were analyzed. Patients with autoimmune disease at delivery were identified by ICD-9 codes for systemic lupus erythematosus (SLE), systemic sclerosis (SSc), rheumatoid arthritis (RA), polymyositis/ dermatomyositis (DM/PM), and juvenile idiopathic arthritis (JIA).

\footnotetext{
"Corresponding Author: HRP Redwood Building, Room T152, 259 Campus Drive, Stanford, CA 94305-5405. jsimard@ stanford.edu phone: +1 6507239680 fax: +1 6507256951 .

Contribution to Authorship:

KDK, JAM, LC, YC, VMK, MD, DKS, GMS, JFS study design; JAM data analysis; KDK, JAM, LC, YC, VMK, MD, DKS, GMS, JFS drafting the manuscript and critical revisions. All authors have seen and approved the final version of the manuscript.

Disclosure of Interests:

The authors report no conflicts of interest. Completed disclosure of interest forms are available to view online as supporting information.

Details of Ethics Approval:

Stanford Institutional Review Board (protocol 24543; 06/26/2018 - 09/30/2018) and the California State Committee for the Protection of Human Subjects (protocol \#12-11-0906; 02/02/2018 - 02/01/2019) approved this study.
} 
Methods: Maternally linked hospital and birth certificate records of 2,481,516 deliveries were assessed (SLE n=2,272, RA n=1,501, SSc n=88, JIA n=187, DM/PM n=38). Multivariable Poisson regression models estimated risk ratios (RRs) for different PTB phenotypes (relative to term deliveries) for each autoimmune disease compared to the general obstetric population adjusting for maternal age, race/ethnicity, body mass index, smoking, education, payer, parity, and prenatal care.

Main Outcome Measures: PTB was assessed overall (20-36 weeks) and by subphenotype: pre-term premature rupture of membranes (PPROM), spontaneous, or medically indicated PTB. Risk of PTB overall and each phenotype was partitioned by gestational age: early (20-31 weeks) and late (32-36 weeks).

Results: Risks for PTB were elevated for each autoimmune disease evaluated: SLE (RR 3.27 95\%CI 3.01-3.56), RA (RR 2.04 95\%CI 1.79-2.33), SSc (RR 3.74 95\%CI 2.51-5.58), JIA (RR 2.23 95\% CI 1.54-3.23), and DM/PM (RR 5.26 95\%CI 3.12-8.89). These elevated risks were observed for the majority of PTB phenotypes as well.

Conclusions: Women with systemic autoimmune diseases appear to have an elevated risk of various PTB phenotypes. Therefore, preconception counseling and close monitoring during pregnancy is crucial.

\section{Tweetable Abstract:}

This study found women with systemic autoimmune diseases have an elevated risk of preterm birth phenotypes.

\section{Keywords}

Preterm Birth; Autoimmune Disease; Connective Tissue Disease; Preterm Premature Rupture of Membranes; Preeclampsia; Eclampsia

\section{Introduction:}

Preterm birth (PTB) substantially impacts maternal and fetal morbidity and mortality $1,2,3$. The earlier a baby is born, the higher the risk for perinatal mortality, long-term adverse neurodevelopmental disabilities, and recurrent health problems that can persist into adulthood $^{2}$. Early-onset preeclampsia is a medical indication for PTB and is associated with substantial maternal morbidity and mortality ${ }^{4}$. There is evidence that medically-indicated PTB increases the risk of neonatal adverse events compared to spontaneous birth at equivalent gestational ages ${ }^{5}$. Thus, better understanding the circumstances of PTB in populations at risk for obstetric complications is needed to optimize care for mother and child. Specifically, several autoimmune rheumatic diseases result in immune system dysregulation and vascular abnormalities that impact obstetric outcomes ${ }^{6}$.

Pregnant women with systemic lupus erythematosus (SLE) are at greater risk of thrombosis, infection, preeclampsia, fetal intra-uterine growth restriction and congenital heart block: complications associated with $\mathrm{PTB}^{7-9}$. Recent data suggest that women with rheumatoid arthritis (RA) are at increased risk for adverse pregnancy events including PTB ${ }^{10,11}$. This increased risk has also been found in women with juvenile idiopathic arthritis (JIA) and 
systemic sclerosis (SSc). ${ }^{12-14}$ There are limited data on obstetric outcomes in patients with dermatomyositis and polymyositis (DM/PM), though a recent population-based study suggests an increased risk of PTB in these patients. However, this study was limited to 17 births ${ }^{15}$.

Although there is some evidence that pregnant women with autoimmune diseases are at greater risk of PTB overall, there is limited understanding of the circumstances of these obstetric complications associated with these conditions. Understanding the occurrence of PTB phenotypes in different autoimmune disease populations can inform clinical care and potentially provide mechanistic insight into such obstetric complications. Accordingly, the goal of this study was to investigate PTB phenotypes in women with autoimmune rheumatic diseases using a large population-based cohort of births in California.

\section{Methods:}

\section{Study population and data:}

Maternally-linked hospital and birth certificate records for all live singleton births (gestational age 20-45 weeks) in California between 2007 and 2011 were included using data provided by the California Office of Statewide Health Planning and Development (OSHPD). California Vital Statistics records linked to statewide hospital discharge data provides information on maternal, pregnancy, and infant characteristics and delivery outcomes. This dataset contains information for nearly every birth in the state of California for the time period assessed. Details of this dataset have been described elsewhere ${ }^{16}$. Stanford Institutional Review Board (protocol 24543) and the California State Committee for the Protection of Human Subjects (protocol \#12-11-0906) approved this study. Patients were not involved in the development to this study and a core outcome set was not used. This study was supported in part by March of Dimes Prematurity Research Center at Stanford and the Stanford Child Health Research Institute

\section{Autoimmune diseases:}

International Classification of Diseases, $9^{\text {th }}$ Revision (ICD-9-CM) diagnosis codes were used to identify women with prevalent autoimmune rheumatic diseases at the time of delivery (SLE, 710.0; SSc, 710.1; RA, 714.0; JIA, 714.3; and DM/PM, 710.3 and 710.4). Women with more than one autoimmune disease diagnosis at delivery were excluded from analyses.

\section{Clinical characteristics:}

Specific demographic and clinical characteristics were obtained from birth certificate records including maternal race/ethnicity, age, pre-pregnancy body mass index (BMI), education, country of birth, parity, gestational age at initiation of prenatal care, payer for delivery, smoking during pregnancy, and gestational age at delivery. The additional maternal co-morbidities of pre-existing hypertension, gestational hypertension, pre-existing diabetes, and gestational diabetes were extracted from the hospital discharge dataset by ICD-9 code. The following codes used were: pre-existing diabetes (Type 1 (250.x1, 250.x3) and Type 2 (250.x0, 250.x2, 648.0)), gestational diabetes (648.8), pre-existing hypertension (401-405, 
642.0, 642.1, 642.2, 642.9), and gestational hypertension (642.3). Women with multiple ICD-9 codes were reclassified to allow for mutually exclusive diabetes groups such that any woman with a gestational diabetes code and a pre-existing diabetes code was reclassified as having pre-existing diabetes.

\section{Preterm birth phenotypes:}

PTB was defined as birth between 20 and $<37$ gestational weeks based on the obstetric estimate and subcategorized as early ( 20 to $<32$ weeks) and late ( 32 to $<37$ weeks). PTB includes delivery due to preterm premature rupture of membranes (PPROM), spontaneous, and medically-indicated subtypes, which we grouped hierarchically using ICD-9 and birth certificate codes. First, births <37 weeks were identified as PPROM (ICD-9 658.1 or birth certificate complication of labor/delivery code 10). Next, spontaneous PTB was identified by diagnosis of premature labor (ICD-9 644) or the use of tocolytics (birth certificate complication/procedure of pregnancy code 28) among those without PPROM. Medicallyindicated PTBs were those induced (ICD-9 procedure 73.0, 73.1, 73.4 or birth certificate complication labor/delivery codes 11,12 ) or delivered by caesarean section (ICD-9 procedure 74 or method of delivery codes $01,11,21,31,02,12,22,32$ ) and not previously identified as PPROM or spontaneous. All births $<37$ weeks not captured by the above groups were considered unclassifiable. We assessed PTB outcomes overall and partitioned into phenotypes: PPROM, spontaneous, and medically-indicated PTB. The percent of medicallyindicated preterm births complicated by preeclampsia/eclampsia, small for gestational age/ intrauterine growth restriction (fetal weight $<10^{\text {th }}$ percentile by sex and/or ICD-9 codes 764.0 and 656.5), and placental abruption (ICD-9 code 641.2) were assessed. These diagnostic codes were not necessarily mutually exclusive.

\section{Preeclampsia:}

Preeclampsia was defined using ICD-9 coding: Mild preeclampsia (642.4), severe preeclampsia/eclampsia $(642.5,642.6)$, and mild or severe preeclampsia or eclampsia superimposed on pre-existing hypertension (642.7). Women with multiple hypertension and/or preeclampsia codes were reclassified as: women with a pre-existing hypertension code and a preeclampsia or eclampsia code were classified as having preeclampsia or eclampsia superimposed on pre-existing hypertension. Women with pre-existing hypertension and gestational hypertension were classified as having pre-existing hypertension. Finally, women with multiple codes for gestational hypertension and preeclampsia or eclampsia were classified according to the most severe disease.

\section{Statistical Analyses:}

Multivariable Poisson regression models estimated risk ratios (RRs) and corresponding 95\% confidence intervals (CIs) for the PTB and preeclampsia outcomes adjusted for maternal age, race/ethnicity, BMI, smoking, education, payer, parity, and prenatal care. Missing covariate data were entered into models as a categorical variable level. Separate models were run for each autoimmune disease and were compared to the general obstetric population after removing mothers with the autoimmune diseases of interest and others such as discoid lupus erythematosus (DLE: ICD-9 695.4) and unspecified connective tissue disease (UCTD: 
ICD-9 710.9) from this referent group. Models for SLE and RA were also assessed stratified on parity.

All data manipulation and analyses were performed using SAS 9.4

\section{Results:}

\section{Baseline characteristics and co-morbid conditions:}

A total of 2,481,516 deliveries were assessed (SLE n=2,272, RA n=1,501, SSc n=88, JIA $\mathrm{n}=187, \mathrm{DM} / \mathrm{PM} \mathrm{n}=38$ ). Demographic and clinical characteristics for all births and by maternal autoimmune disease can be found in Table 1. Ninety percent or greater of women in all groups initiated prenatal care in the first five months of pregnancy and were nonsmokers. Women with JIA were more likely to be nulliparous (64.7\%). With the exception of women with SLE, those with autoimmune diseases were more likely to be nonHispanic White. Women with autoimmune diseases had more pre-existing hypertension, pregnancy-induced hypertension, pre-existing diabetes and gestational diabetes, though the latter was comparable for SLE and all births (7.22\% vs 7.17\%) (Table S1). Missing data (\%) were as follows for SLE, SSc, RA, JIA, DM/PM, All Births, respectively, for race/ethnicity $(2.6,3.4,2.3,0.5,2.6,1.5)$, pre-pregnancy BMI $(7.4,6.8,6.0,2.7,2.6,8.2)$, education (4.1, $4.6,3.6,2.7,7.9,3.3)$, parity $(-,-, 0.1,-,-, 0.1)$, prenatal care $(2.2,3.4,1.6,1.6,2.6,1.9)$, payer $(0.3,-, 0.1,-,-, 0.2)$, smoking $(1.1,1.1,0.9,1.6,-, 1.5)$, and foreign-born $(<0.1,0.0$, $0.0,0.0,0.0,0.1)$.

\section{Preterm birth phenotypes:}

Each of the autoimmune diseases had an increased risk of PTB compared to the general obstetric population (Figure 1). The distribution of gestational age at birth for each autoimmune disease can be found in Figure 2. The increased risk was observed in the majority of PTB phenotypes for each autoimmune disease. Women with SLE had a higher risk for early overall, medically indicated, and spontaneous PTB compared to the corresponding late PTB phenotypes. Women with DM/PM and SSc trended toward a similar pattern of comparably higher risk for many early compared to late PTB phenotypes. However, owing to limited numbers of women in these groups, RRs are less precise across phenotypes. Conversely, the RR was higher for many late PTB phenotypes for women with RA and JIA compared to corresponding early PTB phenotypes, with the exception of spontaneous PTB for women with JIA (Table 2). To account for possible over-fitting, the models were re-run with few adjustments (age, race, and smoking status only) and there was a minimal difference in absolute numbers or calculated RRs for PTB overall and by gestational age (Table S2) or for PTB phenotypes (data not shown). Preeclampsia/eclampsia, small for gestational age/intrauterine growth restriction, and placental abruption complicated medically-indicated preterm births with the following frequencies: $21.4 \%, 16.2 \%$, and $3.9 \%$, respectively.

\section{Preeclampsia:}

As preeclampia/eclampsia are major indications for early delivery, we assessed the RR of these complications by autoimmune disease (Table S3) ${ }^{17}$. The RR of any form of 
preeclampsia was significantly elevated for each autoimmune disease investigated. Risk of severe preeclampsia was particularly high for women with SLE (RR 4.5 95\% CI 3.7-5.4) and with SSc (RR 6.1 95\% CI 2.7-13.5). The risk of preeclampsia superimposed on preexisting HTN was elevated for all autoimmune diseases.

\section{Outcomes stratified on parity:}

Stratifying by parity made little difference when assessing PTB risk overall and early and late PTB phenotypes for RA and SLE (Table S4). However, multiparous women with SLE showed a higher risk for preeclampsia compared to nulliparous women (Table S5). This pattern was also observed for women with RA, for preeclampsia overall, and for mild preeclampsia.

\section{Discussion}

\section{Main Findings:}

Risks of PTB overall and for most PTB phenotypes were increased for all autoimmune rheumatic diseases evaluated in this population-based study of over two million singleton live births. Generally, women with SLE, SSc, and DM/PM had higher risks of PTB at earlier gestational ages, i.e., $<32$ weeks. Conversely, the elevated risk of PTB phenotypes for women with JIA and RA was most prominent at later gestational ages. Medically-indicated early (20-31 weeks) PTB was particularly elevated for women with SLE, potentially owing to the high risk of severe preeclampsia ${ }^{18}$.

\section{Strengths and Limitations:}

This is one of the largest studies investigating PTB outcomes in women with autoimmune diseases in the United States. Unique to this study is the evaluation of pregnancy outcomes in a variety of autoimmune diseases and a focus on multiple PTB phenotypes - phenotypes that likely have complex and differing underlying etiologies. Our study captured a substantial number of deliveries in women with rare diseases (DM/PM and SSc).. However, PTB risks should be interpreted with caution given the small numbers in these groups. Finally, the years assessed were confined to the modern obstetric and rheumatologic management era, so changes in medical practices are unlikely to greatly influence our findings.

Limitations include the use of ICD-9 codes in these administrative data to identify women with prevalent autoimmune diseases which may result in misclassification. Validation studies have yielded variable results. The positive predictive value (PPV) for the maternal diagnosis of SLE has been shown to be $\geq 93 \%$ in administrative data but only 50-60\% using physician billing and hospital discharge data ${ }^{19,20}$. The PPV for the outpatient SSc ICD-9 code is reported at $76 \%$ and $95 \%$ for the inpatient diagnosis of $\mathrm{DM}^{21,22}$. The PPV for an RA ICD-9 code ranges from $34 \%$ to $97 \%$, depending on such factors as specialist and number of patient visits ${ }^{23}$. Finally, data from a managed care population confirmed the diagnosis in $69 \%$ of individuals with an associated JIA ICD- $9 \operatorname{code}^{24}$. As the number of patients with autoimmune diseases is relatively small, we would anticipate that misclassification would be 
minor and the inclusion of such misclassified patients in the general obstetric population would minimally influence the results.

Pregnancy outcomes could not be validated and data are unavailable regarding prior pregnancy complications, autoimmune disease activity, medication use, autoantibody status, and organ-specific manifestations of autoimmune diseases; all of which may impact obstetric outcomes ${ }^{25,26}$. The PPV of gestational age at delivery recorded on birth certificates has been found to be $98-100 \%{ }^{27}$. Anti-phospholipid antibody syndrome is a hypercoagulable state that can result in obstetric complications and frequently co-occurs with autoimmune disease, particularly, SLE. However, information on this diagnosis is unavailable as there is no associated ICD- $9 \operatorname{code}^{28}$. We did not make adjustments by parity for diseases other than RA and SLE due to the number of deliveries assessed.

In the case of autoimmune diseases, it is unknown if diagnoses obtained around delivery over- or underestimate true disease, or whether the misclassification is non-differential ${ }^{29}$. We also found multiparous RA and SLE patients were at increased risk of preeclampsia compared to nulliparous mothers. This is contrary to data from the general population, however this could be an artifact of how prevalent maternal diagnoses were defined as described above ${ }^{30}$.

Interpretation (in light of other literature)

Although there is evidence that the risk for PTB overall is increased in women with autoimmune diseases, the risks for specific phenotypes of PTB have not been clearly defined $^{8,10-15,24-26,31}$. Nationwide Inpatient Sample (NIS) has been used to assess birth outcomes in women with autoimmune diseases. These studies suggest an increased risk for PTB in SLE but less definitive findings or no evidence for PPROM in women with RA, SSc, and DM/PM ${ }^{7,8,32,33}$. Furthermore, those analyses did not account for parity, which is an important factor when evaluating adverse pregnancy outcomes such as preeclampsia ${ }^{34}$. This study accounted for parity and autocorrelation.

A recent small study showed increased medically-indicated compared to spontaneous PTBs in women with SLE. Women with RA had elevated spontaneous compared to medicallyindicated PTBs ${ }^{35}$. We found the RR for spontaneous PTB phenotypes was similar to that of medically-indicated PTB overall for patients with SLE and for patients with RA.

A large Swedish and Danish population based study of pregnancy outcomes in RA found an elevated risk of PTB that was similar for both early and late gestations ${ }^{10}$. However, it is difficult to compare these data to this study as there are differences in maternal characteristics, disease activity, and medication use in these two populations ${ }^{11,36}$.

Population-based studies have shown an increased risk of PTB overall and spontaneous PTB in women with JIA ${ }^{13,37}$. These studies did not evaluate the breadth of PTB phenotypes. Our findings of increased risk of PTB overall, medically-indicated PTB, and preeclampsia in women with JIA are in agreement with a recent Swedish population-based study ${ }^{14}$. However, this study did not evaluate PPROM and it is difficult to compare our results as we could not separate women with pediatric JIA only as was done in the Swedish study. 
The literature describing PTB phenotypes in women with SSc and DM/PM is limited. An Italian multicenter study found no increased risk of PPROM, but an increased risk of early and late PTB (OR 2.17 vs 2.42) in SSc women ${ }^{12}$. We observed a higher risk for early compared to late PTB (RR 9.4 vs. 3.3). Due to the differences in populations, it is difficult to make direct comparisons. Consistent with our results, an Australian population-based study found elevated risks of PTB overall and medically-indicated and spontaneous PTB phenotypes in women with $\mathrm{SSc}^{15}$. For DM/PM, a recent NIS study did not find an increased risk of PPROM, however other PTB phenotypes were not assessed ${ }^{33}$.

We observed increased risks of PTB overall and many PTB phenotypes, particularly at early gestational ages, for women with SLE, SSc, and DM/PM. The observation of a higher risk of delivery at early gestational ages for some diseases may be secondary to bias originating from the depletion of susceptible individuals. However, it is possible that there are mechanistic underpinnings for this pattern of findings. Women with SLE, SSc, and DM/PM have a prominent Type I interferon (IFN) signature that correlates with disease activity and has been implicated in disease pathogenesis ${ }^{38-41}$. There is evidence that pregnant women with SLE who subsequently develop preeclampsia have markedly elevated levels of IFNalpha early in pregnancy compared to SLE women without preeclampsia. Further in vitro work suggests that the antiangiogenic properties of IFN-alpha may lead to poor placentation, potentially contributing to the pathogenesis of preeclampsia ${ }^{42}$. Disordered placentation and abnormal angiogenesis have been associated with PTB, including spontaneous PTB ${ }^{43}$. These findings provide a potential mechanism for the increased risk of some PTB phenotypes for women with SSc, DM/PM, and SLE observed in our study, however further research is needed ${ }^{43-45}$.

\section{Conclusion}

These results indicate that women with systemic autoimmune rheumatic diseases have an elevated risk of PTB phenotypes. Preconception counseling and close monitoring during pregnancy is important. The risk of PTB outcomes was elevated for all autoimmune conditions, however certain diseases had higher risks of specific PTB phenotypes, patterns that may provide insight into the underlying mechanism of these obstetric complications.

\section{Supplementary Material}

Refer to Web version on PubMed Central for supplementary material.

\section{Acknowledgements:}

Funding

This study was supported in part by March of Dimes Prematurity Research Center at Stanford and the Stanford Child Health Research Institute; KDK is funded by the NIH T32 Training Program in Adult and Pediatric Rheumatology 2T32AR050942-11; JFS is funded by NIH NIAMS K01-AR066878.

\section{References:}

1. Goldenberg RL, Culhane JF, Iams JD, Romero R. Epidemiology and causes of preterm birth. Lancet. 2008;371(9606):75-84. [PubMed: 18177778] 
2. Saigal S, Doyle LW. An overview of mortality and sequelae of preterm birth from infancy to adulthood. Lancet. 2008;371(9608):261-269. [PubMed: 18207020]

3. Sibai BM. Diagnosis and management of gestational hypertension and preeclampsia. Obstet Gynecol. 2003;102(1):181-192. [PubMed: 12850627]

4. Lisonkova S, Sabr Y, Mayer C, Young C, Skoll A, Joseph KS. Maternal morbidity associated with early-onset and late-onset preeclampsia. Obstet Gynecol. 2014;124(4):771-781. [PubMed: 25198279]

5. Kurkinen-Raty M, Koivisto M, Jouppila P. Preterm delivery for maternal or fetal indications: maternal morbidity, neonatal outcome and late sequelae in infants. BJOG. 2000;107(5):648-655. [PubMed: 10826581]

6. Ostensen M, Cetin I. Autoimmune connective tissue diseases. Best Pract Res Clin Obstet Gynaecol. 2015;29(5):658-670. [PubMed: 25891380]

7. Clowse ME, Jamison M, Myers E, James AH. A national study of the complications of lupus in pregnancy. Am J Obstet Gynecol. 2008;199(2):127 e121-126.

8. Chakravarty EF, Nelson L, Krishnan E. Obstetric hospitalizations in the United States for women with systemic lupus erythematosus and rheumatoid arthritis. Arthritis Rheum. 2006;54(3):899-907. [PubMed: 16508972]

9. Lateef A, Petri M. Systemic Lupus Erythematosus and Pregnancy. Rheum Dis Clin North Am. 2017;43(2):215-226. [PubMed: 28390564]

10. Norgaard M, Larsson H, Pedersen L, Granath F, Askling J, Kieler H et al. Rheumatoid arthritis and birth outcomes: a Danish and Swedish nationwide prevalence study. J Intern Med. 2010;268(4): 329-337. [PubMed: 20456595]

11. Langen ES, Chakravarty EF, Liaquat M, El-Sayed YY, Druzin ML. High rate of preterm birth in pregnancies complicated by rheumatoid arthritis. Am J Perinatol. 2014;31(1):9-14. [PubMed: 23359233]

12. Taraborelli M, Ramoni V, Brucato A, Airo P, Bajocchi G, Bellisai F et al. Brief report: successful pregnancies but a higher risk of preterm births in patients with systemic sclerosis: an Italian multicenter study. Arthritis Rheum. 2012;64(6):1970-1977. [PubMed: 22213060]

13. Ehrmann Feldman D, Vinet E, Bernatsky S, Duffy C, Hazel B, Meshefedjian G et al. Birth Outcomes in Women with a History of Juvenile Idiopathic Arthritis. J Rheumatol. 2016;43(4): 804-809. [PubMed: 26834215]

14. Remaeus K, Johansson K, Askling J, Stephansson O. Juvenile onset arthritis and pregnancy outcome: a population-based cohort study. Ann Rheum Dis. 2017;76(11):1809-1814. [PubMed: 28663309]

15. Chen JS, Roberts CL, Simpson JM, March LM. Pregnancy Outcomes in Women With Rare Autoimmune Diseases. Arthritis Rheumatol. 2015;67(12):3314-3323. [PubMed: 26434922]

16. Lyndon A, Lee HC, Gilbert WM, Gould JB, Lee KA. Maternal morbidity during childbirth hospitalization in California. J Matern Fetal Neonatal Med. 2012;25(12):2529-2535. [PubMed: 22779781]

17. Turner JA. Diagnosis and management of pre-eclampsia: an update. Int J Womens Health. 2010;2:327-337. [PubMed: 21151680]

18. Simard JF, Arkema EV, Nguyen C, Svenungsson E, Wikstrom AK, Palmsten K. et al. Early-onset Preeclampsia in Lupus Pregnancy. Paediatr Perinat Epidemiol. 2017;31(1):29-36. [PubMed: 27943386]

19. Moores KG, Sathe NA. A systematic review of validated methods for identifying systemic lupus erythematosus (SLE) using administrative or claims data. Vaccine. 2013;31 Suppl 10:K62-73. [PubMed: 24331075]

20. Andrade SE, Scott PE, Davis RL, Li DK, Getahun D, Cheetham T C et al. Validity of health plan and birth certificate data for pregnancy research. Pharmacoepidemiol Drug Saf. 2013;22(1):7-15. [PubMed: 22753079]

21. Kwa MC, Ardalan K, Laumann AE, Nardone B, West DP, Silverberg JI. Validation of International Classification of Diseases Codes for the Epidemiologic Study of Dermatomyositis. Arthritis Care Res (Hoboken). 2017;69(5):753-757. [PubMed: 27564726] 
22. Valenzuela A, Yaqub A, Fiorentino D, Krishnan E, Chung L. Validation of the ICD-9-CM code for systemic sclerosis using updated ACR/EULAR classification criteria. Scand J Rheumatol. 2015;44(3):253-255. [PubMed: 25744697]

23. Chung CP, Rohan P, Krishnaswami S, McPheeters ML. A systematic review of validated methods for identifying patients with rheumatoid arthritis using administrative or claims data. Vaccine. 2013;31 Suppl 10:K41-61. [PubMed: 24331074]

24. Harrold LR, Salman C, Shoor S, Curtis JR, Asgari MM, Gelfand JM et al. Incidence and prevalence of juvenile idiopathic arthritis among children in a managed care population, 19962009. J Rheumatol. 2013;40(7):1218-1225. [PubMed: 23588938]

25. Buyon JP, Kim MY, Guerra MM, Laskin CA, Petri M, Lockshin MD et al. Predictors of Pregnancy Outcomes in Patients With Lupus: A Cohort Study. Ann Intern Med. 2015;163(3):153-163. [PubMed: 26098843]

26. Tedeschi SK, Guan H, Fine A, Costenbader KH, Bermas B. Organ-specific systemic lupus erythematosus activity during pregnancy is associated with adverse pregnancy outcomes. Clin Rheumatol. 2016;35(7):1725-1732. [PubMed: 27166627]

27. Dietz PM, Bombard JM, Hutchings YL Gauthier JP, Gambatese MA, Ko JY et al. Validation of obstetric estimate of gestational age on US birth certificates. Am J Obstet Gynecol. 2014;210(4): 335 e331-335 e335.

28. Abrahams VM, Chamley LW, Salmon JE. Emerging Treatment Models in Rheumatology: Antiphospholipid Syndrome and Pregnancy: Pathogenesis to Translation. Arthritis Rheumatol. 2017;69(9):1710-1721. [PubMed: 28445926]

29. MacDonald SC, Hernan MA, McElrath TF, Hernandez-Diaz S. Assessment of recording bias in pregnancy studies using health care databases: An application to neurologic conditions. Paediatr Perinat Epidemiol. 2018;32(3):281-286. [PubMed: 29569366]

30. Miranda ML, Edwards SE, Myers ER. Adverse birth outcomes among nulliparous vs. multiparous women. Public Health Rep. 2011;126(6):797-805. [PubMed: 22043095]

31. Wei S, Lai K, Yang Z, Zeng K. Systemic lupus erythematosus and risk of preterm birth: a systematic review and meta-analysis of observational studies. Lupus. 2017;26(6):563-571. [PubMed: 28121241]

32. Chakravarty EF, Khanna D, Chung L. Pregnancy outcomes in systemic sclerosis, primary pulmonary hypertension, and sickle cell disease. Obstet Gynecol. 2008;111(4):927-934. [PubMed: 18378753]

33. Kolstad KD, Fiorentino D, Li S, Chakravarty EF, Chung L. Pregnancy outcomes in adult patients with dermatomyositis and polymyositis. Semin Arthritis Rheum. 2017.

34. Hernandez-Diaz S, Toh S, Cnattingius S. Risk of pre-eclampsia in first and subsequent pregnancies: prospective cohort study. BMJ. 2009;338:b2255.

35. Eudy AM, Jayasundara M, Haroun T, Neil L, James AH, Clowse MEB. Reasons for cesarean and medically indicated deliveries in pregnancies in women with systemic lupus erythematosus. Lupus. 2017:961203317720525.

36. de Man YA, Hazes JM, van der Heide H, Willemsen SP, de Groot CJ, Steegers E A et al. Association of higher rheumatoid arthritis disease activity during pregnancy with lower birth weight: results of a national prospective study. Arthritis Rheum. 2009;60(11):3196-3206. [PubMed: 19877045]

37. Chen JS, Ford JB, Roberts CL, Simpson JM, March LM. Pregnancy outcomes in women with juvenile idiopathic arthritis: a population-based study. Rheumatology (Oxford). 2013;52(6):1119_ 1125. [PubMed: 23382363]

38. Feng X, Wu H, Grossman JM, Hanvivadhanakul P, FitzGerald JD, Park GS et al. Association of increased interferon-inducible gene expression with disease activity and lupus nephritis in patients with systemic lupus erythematosus. Arthritis Rheum. 2006;54(9):2951-2962. [PubMed: 16947629]

39. Crow MK. Type I interferon in the pathogenesis of lupus. J Immunol. 2014;192(12):5459-5468. [PubMed: 24907379] 
40. Walsh RJ, Kong SW, Yao Y, Jallal B, Kiener PA, Pinkus JL et al. Type I interferon-inducible gene expression in blood is present and reflects disease activity in dermatomyositis and polymyositis. Arthritis Rheum. 2007;56(11):3784-3792. [PubMed: 17968926]

41. Wu M, Assassi S. The role of type 1 interferon in systemic sclerosis. Front Immunol. 2013;4:266. [PubMed: 24046769]

42. Andrade D, Kim M, Blanco LP, Karumanchi SA, Koo GC, Redecha P et al. Interferon-alpha and angiogenic dysregulation in pregnant lupus patients who develop preeclampsia. Arthritis Rheumatol. 2015;67(4):977-987. [PubMed: 25603823]

43. Straughen JK, Kumar P, Misra VK. The effect of maternal soluble FMS-like tyrosine kinase 1 during pregnancy on risk of preterm delivery. J Matern Fetal Neonatal Med. 2012;25(10):18791883. [PubMed: 22348624]

44. Spencer RN, Carr DJ, David AL. Treatment of poor placentation and the prevention of associated adverse outcomes--what does the future hold? Prenat Diagn. 2014;34(7):677-684. [PubMed: 24799349]

45. Kim YM, Chaiworapongsa T, Gomez R, Bujold E, Yoon BH, Rotmensch S et al. Failure of physiologic transformation of the spiral arteries in the placental bed in preterm premature rupture of membranes. Am J Obstet Gynecol. 2002;187(5):1137-1142. [PubMed: 12439491] 


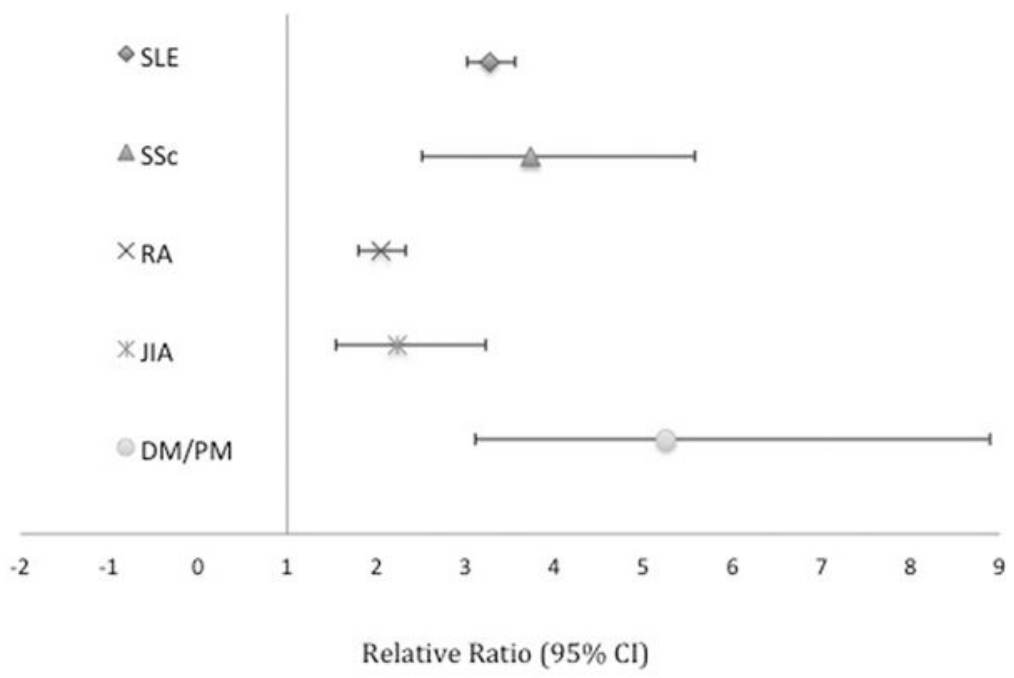

Figure 1. Adjusted risk ratios for preterm births (20-36 weeks) by autoimmune disease. Adjusted for pre-pregnancy BMI, race/ethnicity, education, payer, parity, prenatal care, smoking, and age. 
A.

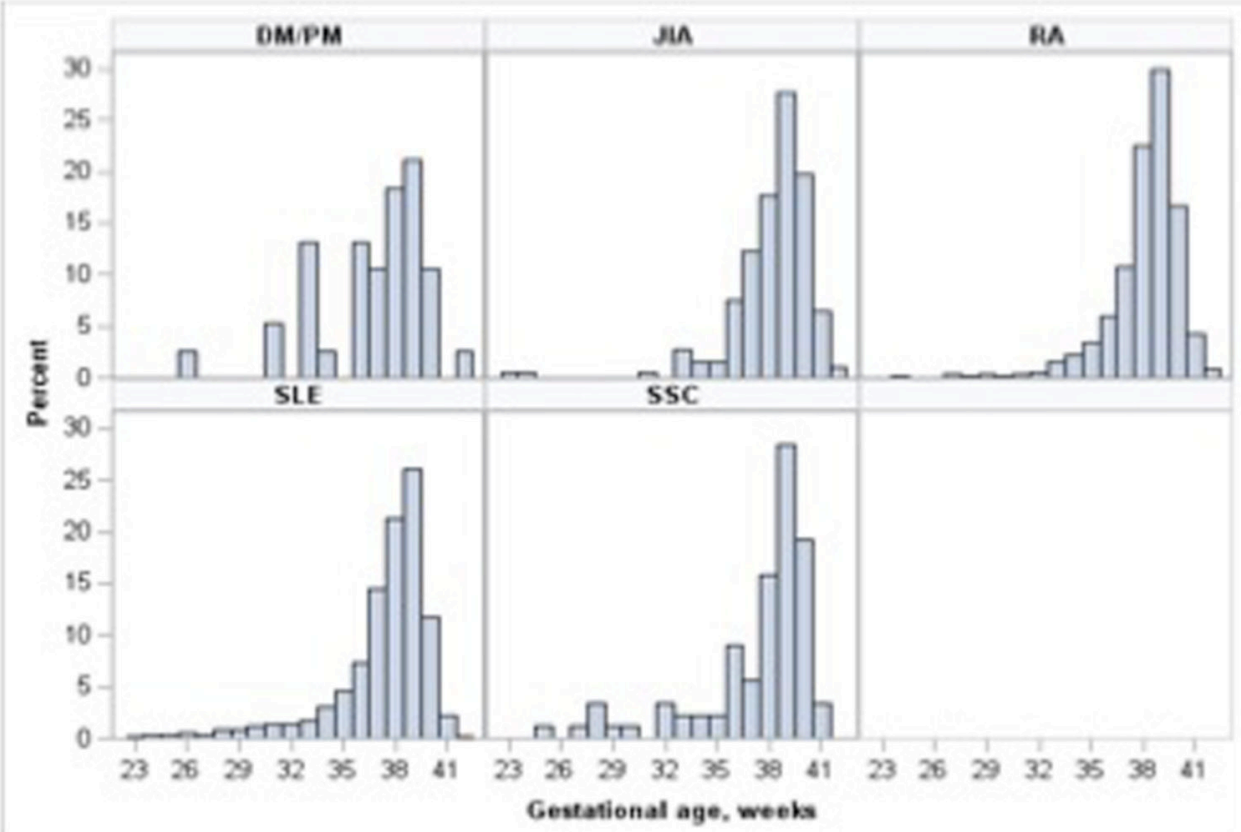

B.

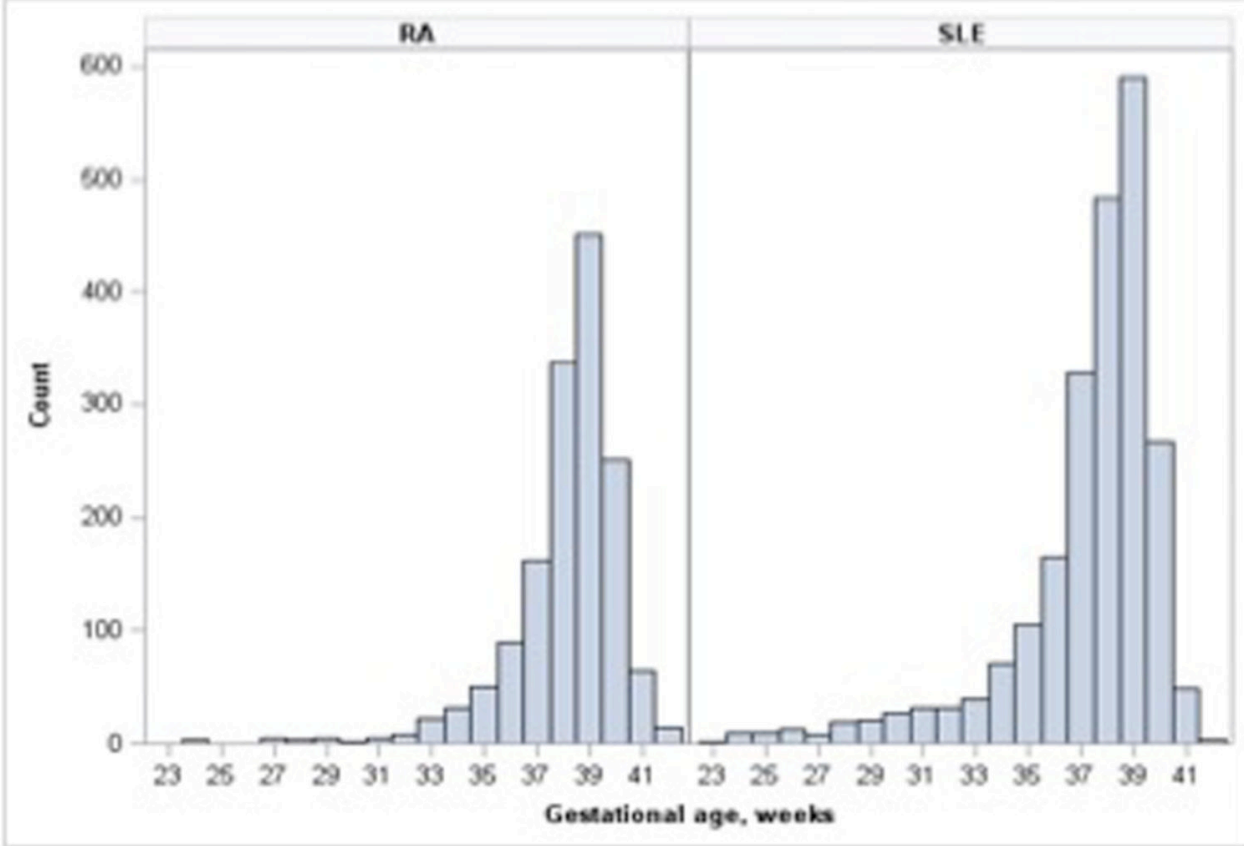

Figure 2.

Distributuion of Gestanational Age at Birth. percent births by gestational age for each autoimmune disease (A). Absolute number of births by gestational age for patients with RA and SLE. Systemic Lupus Erythematosus =SLE, Systemic Sclerosis=SSc, Rheumatoid Arthrits=RA, Polymyositis/Dermatomyositis=DM/PM, Juvenile ldiopathic Arthritis =JIA, 


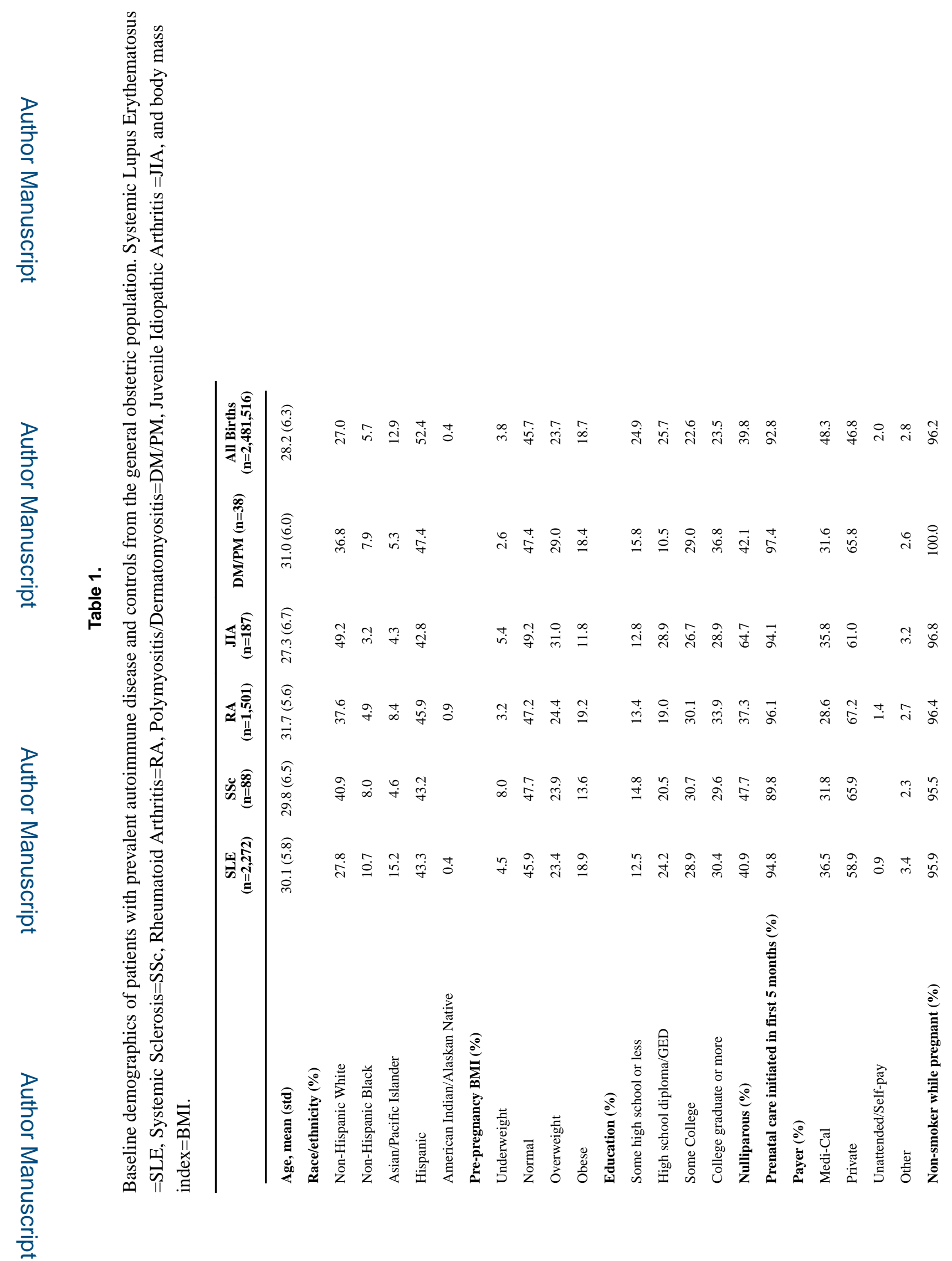

BJOG. Author manuscript; available in PMC 2021 January 01. 


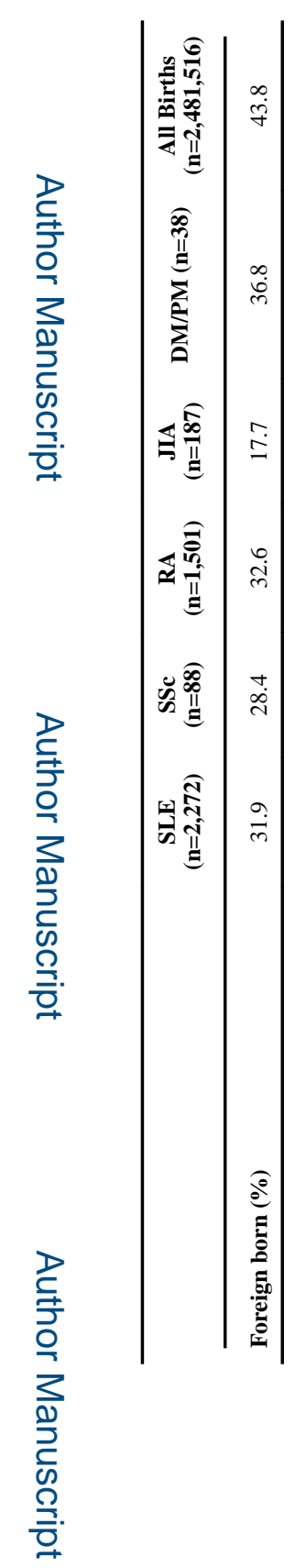

로을

BJOG. Author manuscript; available in PMC 2021 January 01. 


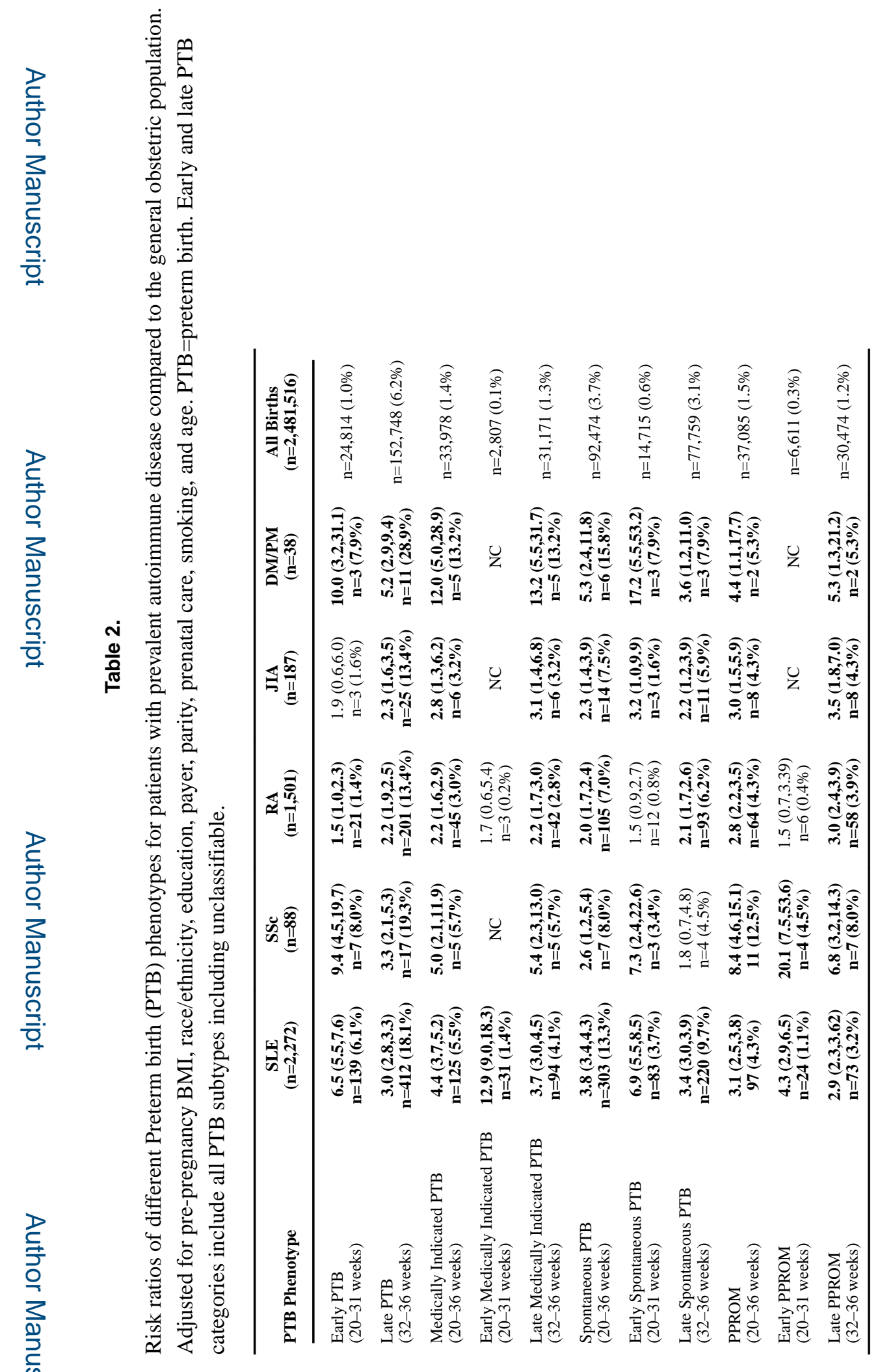

BJOG. Author manuscript; available in PMC 2021 January 01. 